\title{
Raman spectroscopy to differentiate between fresh tissue samples of glioma and normal brain: a comparison with 5-ALA-induced fluorescence-guided surgery
}

\author{
Laurent J. Livermore, DPhil, ${ }^{1,3}$ Martin Isabelle, PhD, ${ }^{4}$ lan M. Bell, DPhil, ${ }^{4}$ Oliver Edgar, BM, BCh, ${ }^{1}$ \\ Natalie L. Voets, PhD, ${ }^{2,6}$ Richard Stacey, FRCS(SN), ${ }^{3}$ Olaf Ansorge, FRCPath, ${ }^{1}$ \\ Claire Vallance, $\mathrm{PhD},{ }^{5}$ and Puneet Plaha, FRCS(SN) $)^{2,3}$ \\ ${ }^{1}$ Nuffield Department of Clinical Neurosciences, and ${ }^{2}$ Nuffield Department of Surgery, University of Oxford, John Radcliffe \\ Hospital, Oxford; ' ${ }^{2}$ Department of Neurosurgery, Oxford University Hospitals NHS Foundation Trust, Oxford; ${ }^{4}$ Renishaw plc, \\ Spectroscopy Products Division, Gloucestershire; ${ }^{5}$ Department of Chemistry, University of Oxford; and ${ }^{6}$ FMRIB Centre, Nuffield \\ Department of Clinical Neurosciences, University of Oxford, John Radcliffe Hospital, Oxford, United Kingdom
}

OBJECTIVE Raman spectroscopy is a biophotonic tool that can be used to differentiate between different tissue types. It is nondestructive and no sample preparation is required. The aim of this study was to evaluate the ability of Raman spectroscopy to differentiate between glioma and normal brain when using fresh biopsy samples and, in the case of glioblastomas, to compare the performance of Raman spectroscopy to predict the presence or absence of tumor with that of 5-aminolevulinic acid (5-ALA)-induced fluorescence.

METHODS A principal component analysis (PCA)-fed linear discriminant analysis (LDA) machine learning predictive model was built using Raman spectra, acquired ex vivo, from fresh tissue samples of 62 patients with glioma and 11 glioma-free brain samples from individuals undergoing temporal lobectomy for epilepsy. This model was then used to classify Raman spectra from fresh biopsies from resection cavities after functional guided, supramaximal glioma resection. In cases of glioblastoma, 5-ALA-induced fluorescence at the resection cavity biopsy site was recorded, and this was compared with the Raman spectral model prediction for the presence of tumor.

RESULTS The PCA-LDA predictive model demonstrated 0.96 sensitivity, 0.99 specificity, and 0.99 accuracy for differentiating tumor from normal brain. Twenty-three resection cavity biopsies were taken from 8 patients after supramaximal resection (6 glioblastomas, 2 oligodendrogliomas). Raman spectroscopy showed 1.00 sensitivity, 1.00 specificity, and 1.00 accuracy for predicting tumor versus normal brain in these samples. In the glioblastoma cases, where 5-ALA-induced fluorescence was used, the performance of Raman spectroscopy was significantly better than the predictive value of 5-ALA-induced fluorescence, which showed 0.07 sensitivity, 1.00 specificity, and 0.24 accuracy $(p=0.0009)$.

CONCLUSIONS Raman spectroscopy can accurately classify fresh tissue samples into tumor versus normal brain and is superior to 5-ALA-induced fluorescence. Raman spectroscopy could become an important intraoperative tool used in conjunction with 5-ALA-induced fluorescence to guide extent of resection in glioma surgery.

https://thejns.org/doi/abs/10.3171/2020.5.JNS20376

KEYWORDS Raman spectroscopy; 5-ALA fluorescence; glioma; oncology

$\mathrm{R}$ ESECTION plays an important role in the treatment of both high- and low-grade gliomas. It is generally accepted that increased extent of resection has a beneficial effect on survival, despite the lack of class I evidence. ${ }^{1,2}$ Maximal resection must not, however, be at the cost of giving the patient a neurological deficit, and the aim of surgery is to achieve maximal resection with- out postoperative deficit. There are many surgical adjuncts available to help the surgeon achieve safe maximum resection, including neuronavigation, intraoperative MRI (iMRI), intraoperative ultrasonography, and fluorescenceguided surgery. ${ }^{3}$ Evidence for the effectiveness of these modalities in maximizing the extent of resection is variable, with randomized trials only available for $\mathrm{iMRI}^{4}$ and

ABBREVIATIONS 5-ALA = 5-aminolevulinic acid; $\mathrm{Cl}=$ confidence interval; $\mathrm{EMSC}=$ extended multiplicative scatter correction; IDH = isocitrate hydrogenase; $\mathrm{IHC}=$ immunohistochemistry; LDA = linear discriminant analysis; $P C=$ principal component; PCA = PC analysis.

SUBMITTED February 12, 2020. ACCEPTED May 22, 2020.

INCLUDE WHEN CITING Published online October 2, 2020; DOI: 10.3171/2020.5.JNS20376. 
5-aminolevulinic acid (5-ALA)-induced fluorescenceguided surgery. ${ }^{5}$ Only 5-ALA-induced fluorescence has been shown to have an effect on patient survival, with the recommendation of its routine use in the 2018 National Institute for Health and Care Excellence (NICE) guidelines for the management of primary brain tumors. ${ }^{6}$ There is evidence, however, that significant amounts of tumor extend beyond the visible fluorescence boundary, with between $25 \%$ and $65 \%$ false-negative rates reported in biopsies taken beyond the visible fluorescent margin. ${ }^{7-11}$

Raman spectroscopy is a vibrational spectroscopy technique that has been applied to the identification of disease in various areas, including neurooncology..$^{12-15}$ By probing the unique molecular vibrations that depend on the composition and structure of samples, Raman spectroscopy provides a wealth of information on a cellular and molecular level of both solid and liquid specimens. The huge advantage of the technique is that it is nondestructive, and no sample preparation is required. This makes it ideal for intraoperative neurosurgical applications to define tumor margins. Raman spectroscopy can accurately differentiate between normal brain, necrosis, and various brain tumor pathologies when applied to either formalin-fixed, paraffin-embedded ${ }^{16}$ or cryopreserved ${ }^{17-26}$ histopathological samples. There has only been one report of intraoperative use of Raman spectroscopy in neurosurgery, which reported the ability to distinguish normal brain from both bulk tumor and infiltrating tumor with an accuracy of $92 \%$, sensitivity of $93 \%$, and specificity of $91 \% .{ }^{27}$

The aims of this study were twofold: 1) evaluate the ability of Raman spectroscopy to differentiate between glioma and normal brain when using fresh biopsy samples, and 2) in the case of glioblastomas, compare the performance of Raman spectroscopy in predicting the presence or absence of tumor with that of 5-ALA-induced fluorescence.

In our center, where appropriate, some patients undergo functional guided resection of glioblastomas ${ }^{28}$ with the aim of achieving a maximal or even supramaximal functional resection when safe to do so (all cases performed by the senior author [P.P.]). In these cases, the resection already goes beyond the visual 5-ALA-induced fluorescence boundary, and thus there are biopsies available from regions of nonfluorescing tissue resection that can be used to evaluate novel tumor-detecting modalities such as Raman spectroscopy.

\section{Methods}

\section{Study Participants and Intraoperative Biopsy}

Ethical approval for the use of human tissue was obtained through the Oxford Brain Bank Research Ethics Committee approval. All tissue used was deemed surplus to diagnostic requirements. A subset of patients who underwent functional guided resection of a glioma, where supramaximal resection was achieved, were included in this study. A $20 \mathrm{mg} / \mathrm{kg}$ oral dose of 5-ALA was given to all patients with suspected glioblastoma on preoperative imaging unless contraindicated. The 5-ALA was given 2 hours before the start of surgery to achieve peak fluorescence at approximately 4-6 hours. ${ }^{29,30}$

Our method of functional guided surgery has been pre- sented elsewhere. ${ }^{28}$ Briefly, we define "functional guided surgery" as resecting tumor up to approximately $1.5-2 \mathrm{~cm}$ beyond the contrast-enhancing boundary on neuronavigation (or the 5-ALA fluorescence boundary, as 5-ALA fluorescence is known to closely correlate with contrast enhancement on $\mathrm{MRI}^{8,11}$ ) or resecting until a functional white fiber tract or functional brain region is reached. If there is no functional brain encountered, then an approximately 1.5 - to $2-\mathrm{cm}$ margin beyond the contrast-enhancing or fluorescing boundary is considered the limit of resection, and a "supramaximal" resection is said to have been achieved. To evaluate the functional boundaries, diffusion tensor imaging is undertaken preoperatively and incorporated with the neuronavigation (Brainlab) to identify the spatial location of white matter tracts relative to tumor; awake craniotomy is performed in most of these cases.

Given the intratumoral heterogeneity of gliomas, we routinely take tumor biopsy samples from different anatomical locations within and at the limit of the tumor resection. The location of each biopsy sample is recorded using the intraoperative neuronavigation system (Fig. 1) and labeled according to its spatial anatomical location. Prior to resection, each sample location is inspected for 5-ALA-induced fluorescence using the blue light source on either the operative microscope (Zeiss OPMI Pentero 900, Carl Zeiss) or endoscope (Karl Storz Hopkins endoscope, Karl Storz Endoscopy) and the result recorded. The degree of fluorescence is reported as "none" (no fluorescence), "weak" fluorescence, or "strong" fluorescence, using the fluorescence grading system proposed by Stummer et al., "who defined visual fluorescence as either "strong" ("solid" or "red") or "weak" ("vague" or "salmon"). Microbiopsy forceps are used to excise the biopsy sample, and the fresh tissue is taken to neuropathology for immediate examination. Figure 2 depicts tumor cavities with different degrees of fluorescence and the corresponding white light images.

\section{Neuropathological Evaluation}

Each biopsy sample, approximately $3 \mathrm{~mm}^{3}$, was cut in half: half was taken straight for Raman analysis with no further preparation, and half was processed as part of the routine diagnostic pathway, undergoing formalin fixation and paraffin embedding. For tumor found to be isocitrate hydrogenase (IDH)-mutated on routine IDH1-R132H immunohistochemistry (IHC), the resection cavity biopsies also underwent IDH1-R132H IHC testing to identify tumor cells within the sample. No tumor carried a rare IDH1 or IDH2 mutation in this study. For tumors diagnosed as IDH-wildtype, the cavity biopsies underwent Ki-67/MIB1 IHC. MIB is an antibody for Ki-67 protein that is expressed in the active phase of the cell cycle; thus, Ki-67/MIB1 is a marker of cell proliferation. ${ }^{31}$ Although not specific for tumor cells, the amount of cell proliferation together with atypical morphology of labeled nuclei in a section gives an indication of the presence of tumor infiltration. With the combination of the standard $\mathrm{H} \& \mathrm{E}$ and the appropriate IHC stain, the neuropathologist categorized the cavity biopsies into three groups (examples illustrated in Fig. 3): 1) tumor = sample is characteristic of tumor throughout (Fig. 3D, H, I, and L); 2) infiltrating tumor = evidence of 

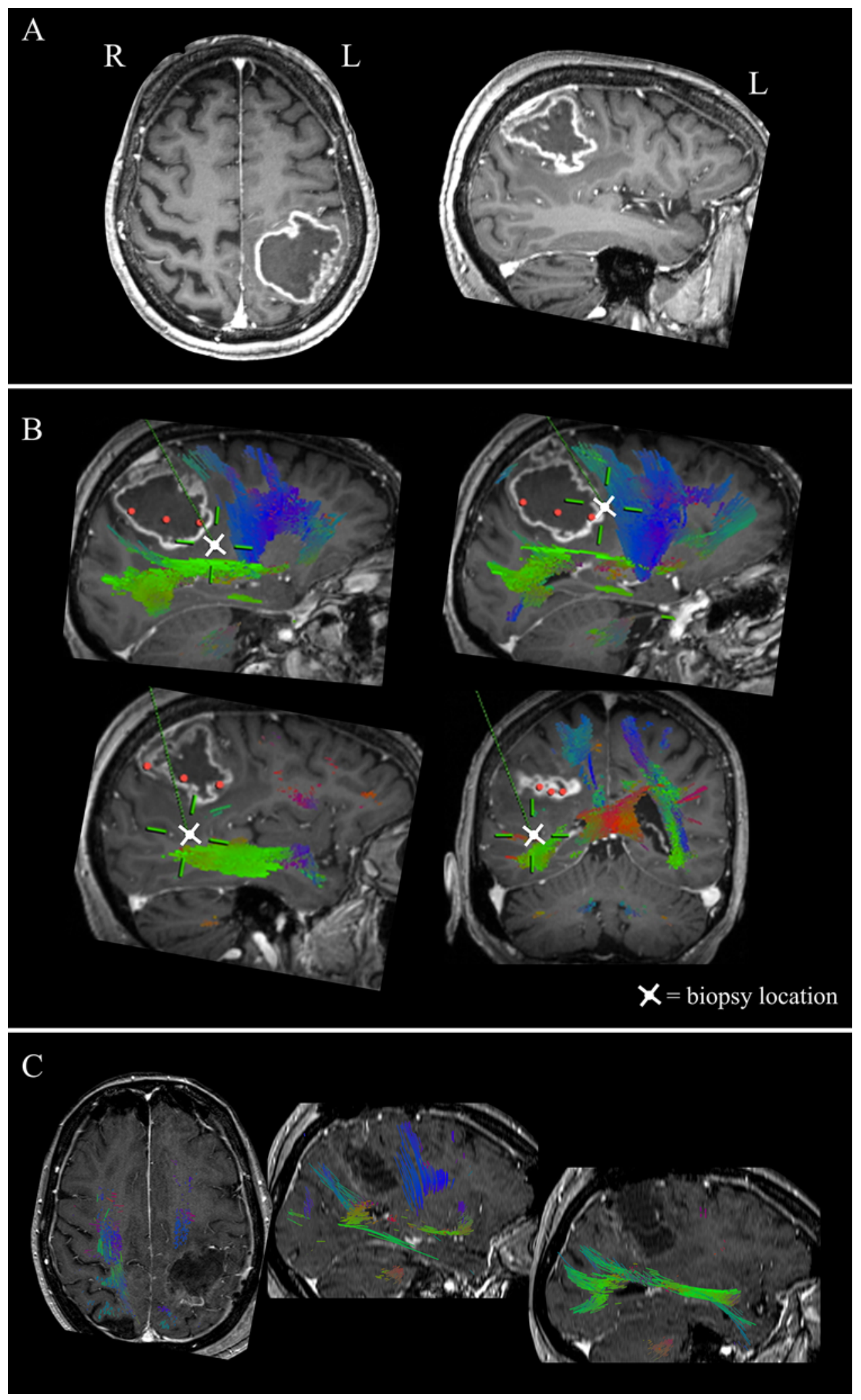

FIG. 1. MRI for supramaximal resection. A: Preoperative MRI demonstrating enhancing tumor consistent with glioblastoma. B: Intraoperative neuronavigation showing the location of supramaximal resection cavity biopsies beyond contrast-enhancing tumor. C: Postoperative MRI demonstrating supramaximal resection up to the white matter tracts. All images are T1-weighted sequences with gadolinium contrast. Colored overlay represents the estimated location of white fiber tracts using diffusion tensor imaging. Figure is available in color online only. 

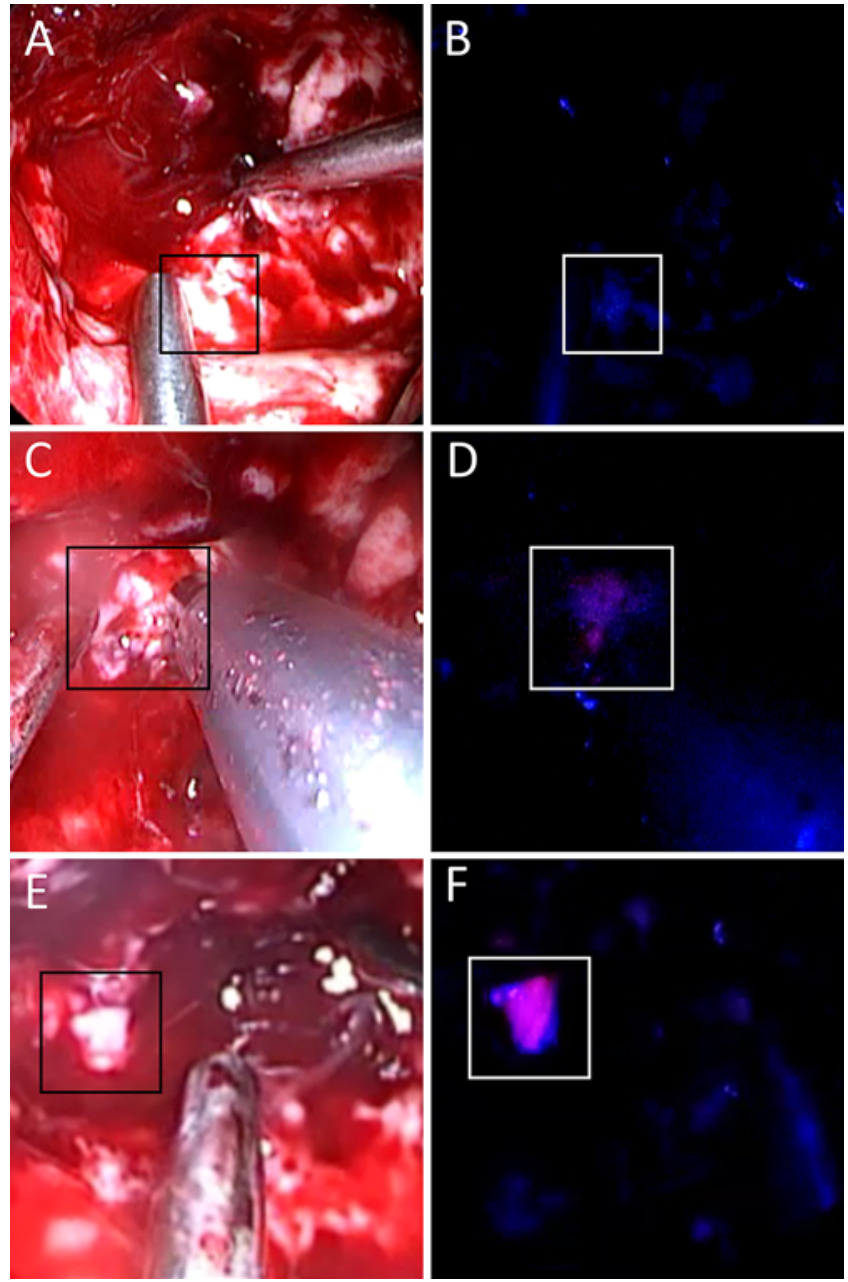

FIG. 2. Examples of fluorescence quantification. Resection cavity biopsy location with no visual fluorescence with blue light (B) and corresponding white light image (A). Resection cavity biopsy location with "weak" fluorescence with blue light (D) and corresponding white light image (C). Example of strong fluorescence $(\mathbf{F})$ and corresponding white light image (E). Outlined quadrants show the location of the biopsy taken. Figure is available in color online only.

tumor cell infiltration into normal white or gray matter (the degree of infiltration can vary; Fig. 3A-C, E-G, J, and $\mathrm{K}$ ); and 3) no tumor cells = histological and IHC features of normal brain with no evidence of tumor.

This histological grading system used is modified from the grading system reported Lau et al., ${ }^{7}$ a paper that aimed to correlate intraoperative fluorescence intensity with the degree of histological cellularity. In the current study, the "low infiltrating" and "moderate infiltrating" grades (grades 1 and 2 in the Lau et al. paper) are grouped into "infiltrating tumor," giving a three-grade system: "no definitive tumor cells," "infiltrating tumor," and "tumor" (Fig. 3).

\section{Normal Fresh Brain Biopsies}

To build a classification model using Raman spectra to differentiate between tumor and normal brain, it was nec- essary to record Raman spectra from several normal fresh brain biopsies. These were acquired from patients undergoing mesial temporal lobectomy for epilepsy, in which a small amount of normal brain is routinely removed as access tissue. The normal brain biopsies, incorporating both gray and white matter, were halved, with half going straight for Raman analysis and half undergoing standard $\mathrm{H} \& \mathrm{E}$ staining and neuropathology evaluation to confirm the presence of normal brain tissue.

\section{Raman Spectroscopy}

Our protocol for the intraoperative rapid analysis of fresh samples has been reported previously. ${ }^{26}$ Briefly, each fresh tissue sample was compressed into a grooved stainless-steel slide and the slide placed in the Raman spectrometer (Renishaw benchtop RA800 series spectrometer, Renishaw plc) equipped with a $\times 50$ objective and a $785-\mathrm{nm}$ excitation laser (outside the excitation or fluorescence ranges of porphyrin IX) that provided 180 $\mathrm{mW}$ of power at the objective. Preliminary experiments showed no effect of porphyrin IX on the Raman spectra. Spectra were obtained from 50 to 200 widely distributed and randomly chosen locations across the smeared sample surface. The number of spectra collected varied depending on the amount of in-focus fresh tissue available for analysis. The exposure time used for each spectrum was 2 seconds.

\section{Development of the Classification Model: "Tumor" Versus "Normal Brain"}

All model development and data analysis were performed using the Renishaw Data Classifier (Renishaw plc) and MATLAB R2015b (MathWorks). The Raman spectral region between 400 and $1850 \mathrm{~cm}^{-1}$ was analyzed, excluding the Raman band of oxygen (1560-1578 $\mathrm{cm}^{-1}$ ). Spectral preprocessing steps included signal-to-noise threshold filter, cosmic ray removal using a standard deviation threshold, third-order polynomial baseline correction, and normalization using extended multiplicative scatter correction (EMSC). ${ }^{32}$

A two-group classification model-tumor versus normal brain -was built using principal component analysis (PCA)-fed linear discriminant analysis (LDA). In this method, PCA is used to reduce the data dimensionality, with ANOVA used to select only those principal components (PCs) that describe a statistically significant difference between the two data sets. An LDA model (supervised machine learning model) is then built from those significant PCs. Model performance was assessed using leave-one-sample-out independent cross-validation to generate model sensitivity and specificity. A binomial distribution hypothesis test was conducted on each sample to ensure that the number of spectra obtained did not significantly influence the model prediction accuracy. The mean spectra and the negative of the second derivative transformation of the mean spectra were calculated using the normalized spectra from each classification group. Using the normalized mean spectra, statistical differences between the Raman peak intensities in each classification group were evaluated using the Wilcoxon rank-sum test. Tenta- 

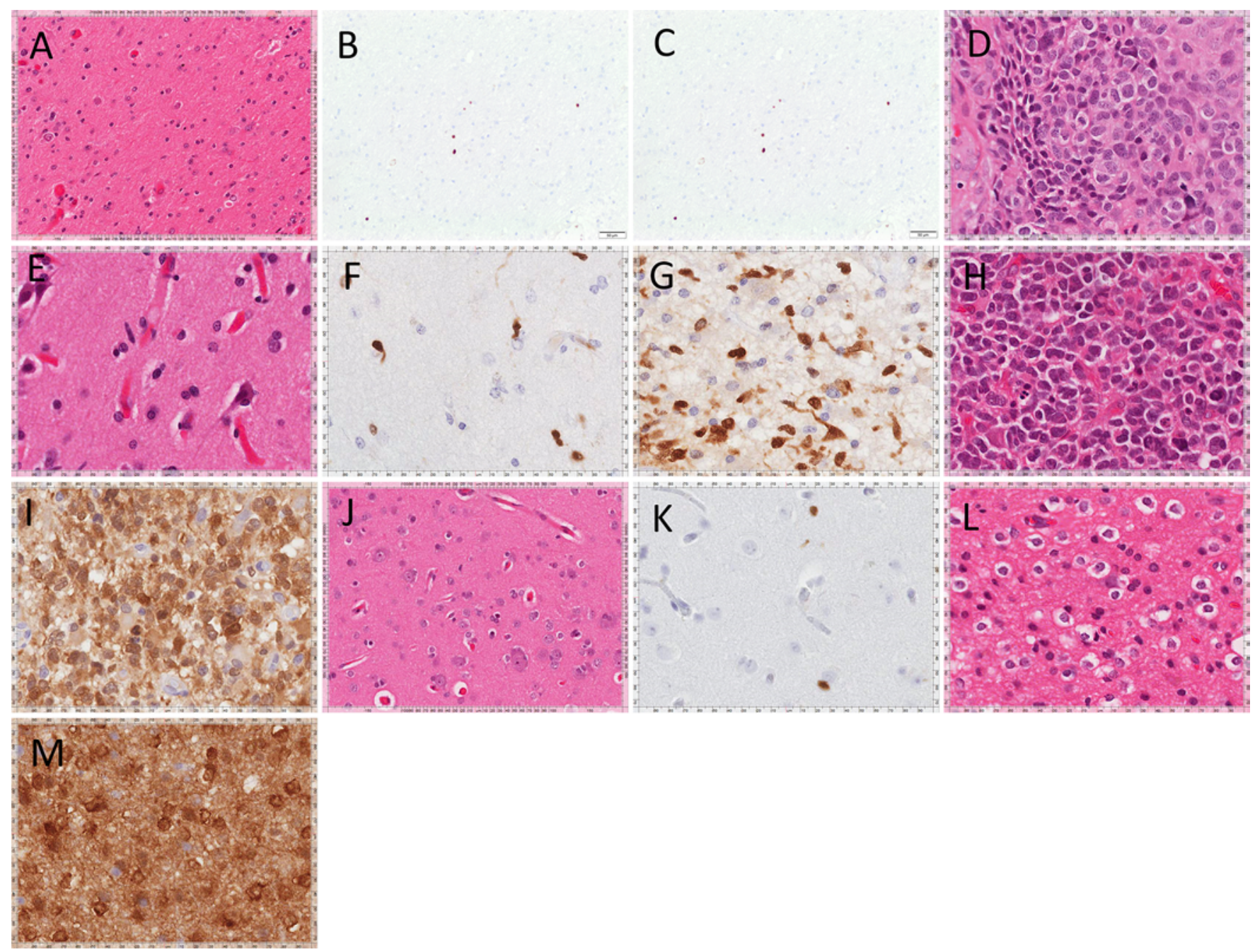

FIG. 3. Histological grading system. A: Glioblastoma-wildtype, infiltrating tumor. H \& E, original magnification $\times 50$. B: Glioblastoma-wildtype, low-density infiltrating tumor. MIB, original magnification $\times 50$. C: Glioblastoma-wildtype, higher-density infiltrating tumor. MIB, original magnification $\times 50$. D: Glioblastoma-wildtype, tumor. H \& E, original magnification $\times 100$. E: Glioblastoma-IDHmutated, infiltrating tumor. $\mathrm{H} \& \mathrm{E}$, original magnification $\times 100$. F: Glioblastoma-IDH-mutated, infiltrating tumor. IDH-R132, original magnification $\times 100$. G: Glioblastoma-IDH-mutated, higher-density infiltrating tumor. IDH-R132, original magnification $\times 100$.

H: Glioblastoma-IDH-mutated, tumor. H \& E, original magnification $\times 100$. I: Glioblastoma-IDH-mutated, tumor. IDH-R132, original magnification $\times 100$. J: Oligodendroglioma, infiltrating tumor. $\mathrm{H} \& \mathrm{E}$, original magnification $\times 100$. K: Oligodendroglioma, infiltrating tumor. IDH-R132, original magnification $\times 100$. L: Oligodendroglioma, tumor. H \& E, original magnification $\times 100$. M: Oligodendroglioma, tumor. IDH-R132, original magnification $\times 100$. Figure is available in color online only.

tive molecular assignment of the identified Raman peaks was performed based on the published literature. ${ }^{17,22,33}$

For the "tumor" group, the input spectra came from the data set of intraoperative fresh tissue spectra collected for another study reported elsewhere. ${ }^{26}$ The "normal brain" group input spectra were taken from the fresh tissue analysis of normal brain samples acquired from epilepsy surgery cases as described above.

\section{Classification of Resection Cavity Spectra and Comparison With 5-ALA-Induced Fluorescence}

Spectra from the resection cavity samples were individually classified into one of the two groups in the built model (individual spectra classification). The percentage of spectra classified into each group for a given biopsy was also calculated, and the group with the greatest number of spectra classified into it was taken to be the resultant overall classification of that biopsy (overall biopsy classification). Values of sensitivity, specificity, and accuracy were calculated in each case, along with $95 \%$ confidence interval (CI) calculations (Clopper-Pearson $\mathrm{CIs}^{34,35}$ ), and compared with similar parameters for 5-ALA-induced fluorescence in the cases of glioblastoma where 5-ALA was used. McNemar's test for related categorical data ${ }^{36,37}$ was used to measure the statistical differences between Raman and 5-ALA for differentiating tumor versus normal brain. 
TABLE 1. PC-LDA model of tumor versus normal brain

\begin{tabular}{cccc}
\hline Classification Group & Sensitivity & Specificity & Accuracy \\
\hline Tumor & 0.96 & 0.99 & 0.99 \\
\hline Normal brain & 0.99 & 0.96 & 0.99 \\
\hline
\end{tabular}

Performance of model using a leave-one-patient-out cross-validation.

\section{Results}

\section{"Tumor" Versus "Normal Brain" Classification Model}

Eleven fresh normal brain biopsies were acquired from 11 patients undergoing temporal lobectomy for epilepsy. Neuropathology review confirmed normal gray and white matter in every case. A total of 1825 spectra of fresh normal brain were used in the model as "normal brain" input spectra, with a mean of 166 spectra per sample. For the "tumor" input spectra, 9799 spectra from 62 fresh tissue samples originating from another study were used. ${ }^{26}$ This included 36 glioblastomas, IDH-wildtype; 10 glioblastomas, IDH-mutant; 7 astrocytomas, IDH-mutant WHO grade III; 4 astrocytomas, IDH-mutant WHO grade II; 2 anaplastic oligodendrogliomas, WHO grade III; and 3 oligodendrogliomas, WHO grade II. The model performed extremely well when assessed using leave-one-patient-out cross-validation, as shown by the high sensitivity and specificity illustrated in Table 1 and Fig. 4. The mean spectra and the negative of the second derivative transformation of the mean spectra for "tumor" and "normal brain" are illustrated in Fig. 5. The difference spectrum (tumor minus normal brain) is also shown in this figure. Lipid peaks are more prominent in tumor compared to normal brain spectra, with dominant peaks at 825, 853, 1087, 1124, and 1305 $\mathrm{cm}^{-1}$. This is also true for peaks representing DNA (517, 885,1206 , and $\left.1342 \mathrm{~cm}^{-1}\right)$. Conversely, normal brain spectra have stronger amino acid peaks $(540,615,635$, and 649 $\mathrm{cm}^{-1}$ ) and protein peaks (1522 and $1553 \mathrm{~cm}^{-1}$, both representing amide III protein) compared with tumor spectra.

\section{Classification of Resection Cavity Spectra and Comparison With 5-ALA-Induced Fluorescence}

Raman analysis of 23 supramaximal resection cavity biopsies was undertaken in a total of 8 patients. The cases and corresponding biopsies are summarized in Table 2. There were 9 biopsies from 3 glioblastoma, IDH-wildtype tumors; 8 biopsies from 3 glioblastoma, IDH-mutant tumors; and 6 biopsies from 2 oligodendrogliomas (1 WHO grade II and 1 WHO grade III). All neuronavigation images documenting the location of the resection cavity biopsies were beyond the area of enhancement on the contrast-enhanced preoperative MRI. The neuropathology assessment categorized 2 biopsies as tumor, 17 as infiltration zone, and 4 as having no tumor cells.

In all 6 glioblastoma cases 5-ALA was used, and all showed strong fluorescence in the main bulk of the tumor. Fluorescence was observed at the site of biopsy in 1 of 17 biopsies (Table 2). For the cases in which 5-ALA was used, the absence of fluorescence was highly specific (specificity $=1.00$, rate of true negatives) - as in all biopsies, where there was no tumor there was also no fluorescence-but

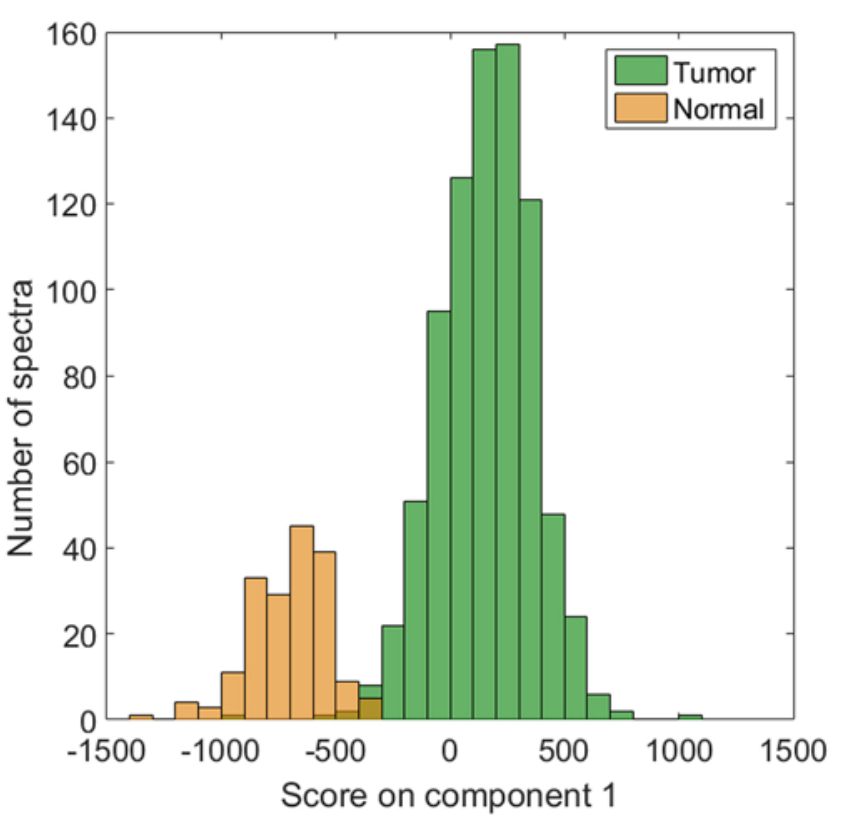

FIG. 4. PC-LDA model of tumor versus normal brain. LDA score histogram. Figure is available in color online only.

poorly sensitive (sensitivity $=0.07$ ), as most cavity biopsies containing tumor did not fluoresce.

Table 3 compares the performance of 5-ALA-induced fluorescence for predicting tumor versus normal brain with the performance of Raman classification models. Raman performance depends on which method is used to classify the biopsy samples into tumor/normal brain groups. Using overall biopsy classification-classifying the biopsy based on the group with the greatest percentage of spectra classified to it- the performance is extremely high, with correct classification of biopsies in every case, although there is a wide $95 \%$ CI range due to the low sample size. This performance is significantly better than 5-ALA-induced fluorescence $\left(\chi^{2}=11.077, \mathrm{p}=0.0009\right)$. Using individual spectra classification, there is still far superior, and statistically significant, performance compared to 5-ALA-induced fluorescence $\left(\chi^{2}=1920.12, p=0.0001\right)$. The performance is also high when oligodendroglioma cases are included. In the 2 oligodendrogliomas 5-ALA was not used, and thus a comparison between Raman spectroscopy and fluorescence is not possible for these cases. It is possible, however, to conclude that Raman spectroscopy is more sensitive than the contrast-enhancing boundary identified on the neuronavigation (both oligodendrogliomas were contrast-enhancing on preoperative MRI).

\section{Discussion}

This preliminary study shows that Raman spectroscopy can accurately differentiate between infiltrating glial tumor and normal brain at the margin of a functionally guided, supramaximal glial tumor resection cavity. Raman spectroscopy is also significantly superior to 5-ALAinduced fluorescence in predicting glioblastoma tumor infiltration versus normal brain at this margin. 

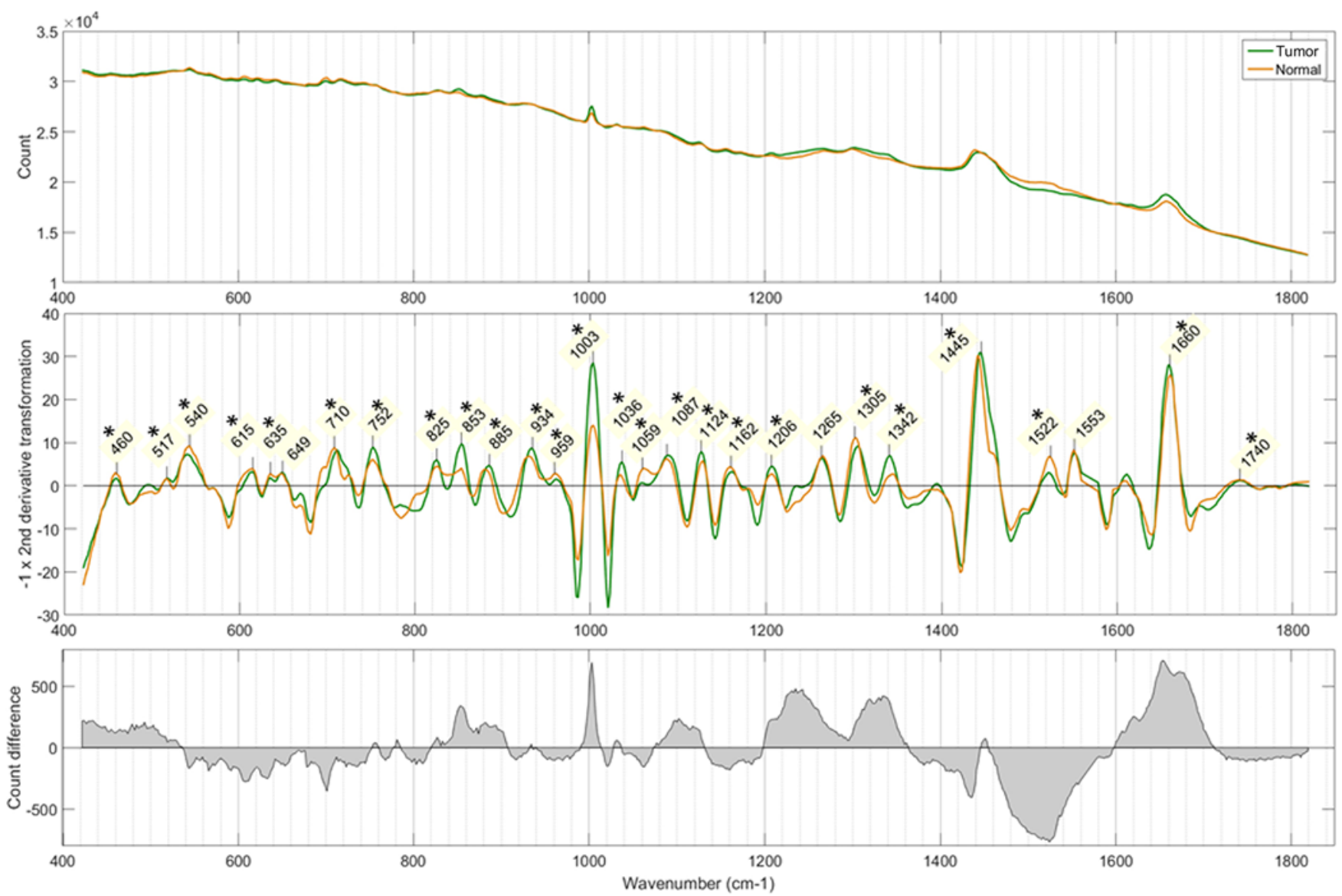

FIG. 5. Tumor and normal brain spectra. Mean spectra (upper), difference spectra (lower), and negative of second derivative transformation of mean spectra for tumor and normal brain after EMSC (center). The wavenumbers of prominent Raman peaks are labeled and the statistical significance $(p<0.01)$ of the difference between tumor and normal brain peak intensities is indicated by an asterisk. Figure is available in color online only.

\section{"Tumor" Versus "Normal Brain" Classification Model}

To build a predictive classification model to classify normal brain and tumor, Raman spectra from fresh tissue samples of the core of 62 different gliomas were used to represent "tumor" and Raman spectra from fresh tissue samples of 11 patients undergoing mesial temporal lobectomy for epilepsy were used to represent "normal brain." The tumor group included a range of different tumor types with the assumption, backed up by evidence in the literature ${ }^{17,22,24}$ (including work carried out by our group ${ }^{26}$ ), that the various tumor types are chemically closer to each other than they are to normal brain tissue. Using independent cross-validation, the resulting model demonstrated excellent performance in classifying tumor versus normal brain spectra (Table 1, Fig. 4). These data are consistent with previous studies that have demonstrated the potential of Raman spectroscopy to differentiate glioma from normal brain tissue using ex vivo formalin-fixed, paraffin-embedded ${ }^{16,26}$ frozen tissue ${ }^{17,20,22,24}$, 26,38 and ex vivo fresh tissue. ${ }^{26,39}$ Moreover, the differences found in this study between the normal brain and tumor spectra, namely dominant lipid and DNA peaks and reduced protein peaks in the tumor spectra, are also consistent with previous reports in the literature. . $^{18,20,22,24}$

\section{Classification of Resection Cavity Spectra and Comparison With 5-ALA-Induced Fluorescence}

In the bulk of the tumor and up to the contrast-enhancing margin seen on image guidance, there is little doubt that 5-ALA-induced fluorescence is a powerful surgical adjunct. The correlation between contrast-enhancing tumor on preoperative MRI and 5-ALA-induced fluorescence is well recognized, ${ }^{8}$ and accounts for increased extent of tumor resection and progression-free survival when using 5-ALA-induced fluorescence-guided surgery. ${ }^{5}$ Fluorescence-guided surgery is conducive to the neurosurgical workflow, only requiring the surgeon to press a button to switch from white to blue light while viewing the tissue through the surgical microscope. There is no need to pause resection or to change instruments as would be the case with a probe-based system. This study focuses on biopsy samples taken at the boundaries of supramaximal resections for glioblastoma cases, beyond the contrast enhancement and 5-ALA-induced fluorescence. Here, the predictive value of 5-ALA is reduced, with literature reporting between $25 \%$ and $65 \%$ false-negative rates in biopsies taken beyond the visual fluorescent margin..$^{71}$ In this study we report an even higher false-negative rate, with only 1 
TABLE 2. Supramaximal resection cases and resection cavity biopsies

\begin{tabular}{|c|c|c|c|c|c|c|c|c|}
\hline \multirow{2}{*}{$\begin{array}{l}\text { Pathology } \\
\text { (WHO grade) }\end{array}$} & \multirow{2}{*}{$\begin{array}{l}\text { Resection } \\
\text { Cavity Biopsy } \\
\text { Location }\end{array}$} & \multirow{2}{*}{$\begin{array}{c}\text { 5-ALA Fluorescence } \\
\text { Seen at Location of } \\
\text { Quadrant }\end{array}$} & \multicolumn{3}{|c|}{$\begin{array}{c}\text { Neuropathology } \\
\text { Assessment }\end{array}$} & \multicolumn{3}{|c|}{$\begin{array}{l}\text { Classification of } \\
\text { Spectra }\end{array}$} \\
\hline & & & $\mathrm{IDH}^{*}$ & MIB-1† & Categorization & No. of Spectrał & Proportion Tumor & Proportion Normal \\
\hline \multirow{2}{*}{ GB-WT } & Anterior & None & & 0 & No tumor cells & 65 & 0.37 & 0.63 \\
\hline & Posterior & None & & $5 \%$ & Infiltrating tumor & 194 & 0.99 & 0.01 \\
\hline \multirow{4}{*}{ GB-WT } & Posterior & None & & $10 \%$ & Infiltrating tumor & 217 & 0.99 & 0.01 \\
\hline & Inferior & Weak & & $5 \%$ & Infiltrating tumor & 163 & 0.96 & 0.04 \\
\hline & Posterior inferior & None & & $10 \%$ & Infiltrating tumor & 160 & 0.92 & 0.08 \\
\hline & Medial & None & & $10 \%$ & Infiltrating tumor & 180 & 0.94 & 0.06 \\
\hline \multirow{3}{*}{ GB-WT } & Anterior & None & & $5 \%$ & Infiltrating tumor & 99 & 1 & 0 \\
\hline & Medial & None & & $5 \%$ & Infiltrating tumor & 160 & 0.95 & 0.05 \\
\hline & Posterior & None & & $5 \%$ & Infiltrating tumor & 122 & 0.99 & 0.01 \\
\hline \multirow{3}{*}{ GB-MUT } & Inferior & None & + & & Tumor & 144 & 1 & 0 \\
\hline & Posterior & None & + & & Infiltrating tumor & 183 & 0.97 & 0.03 \\
\hline & Superior & None & + & & Infiltrating tumor & 105 & 1 & 0 \\
\hline \multirow{2}{*}{ GB-MUT } & Anterior & None & + & & Tumor & 107 & 0.97 & 0.03 \\
\hline & Posterior & None & - & & No tumor cells & 206 & 0.23 & 0.77 \\
\hline \multirow{3}{*}{ GB-MUT } & Inferior & None & + & & Infiltrating tumor & 86 & 0.94 & 0.06 \\
\hline & Posterior & None & - & & No tumor cells & 183 & 0.31 & 0.69 \\
\hline & Medial & None & + & & Infiltrating tumor & 108 & 1 & 0 \\
\hline \multirow{3}{*}{ Oligo (III) } & Posterior & Not used & + & & Infiltrating tumor & 72 & 0.97 & 0.03 \\
\hline & Inferior lateral & Not used & + & & Infiltrating tumor & 42 & 0.83 & 0.17 \\
\hline & Superior & Not used & + & & Infiltrating tumor & 186 & 0.97 & 0.03 \\
\hline \multirow{3}{*}{ Oligo (II) } & Frontal & Not used & + & & Infiltrating tumor & 78 & 0.97 & 0.03 \\
\hline & Temporal & Not used & - & & No tumor cells & 170 & 0.41 & 0.59 \\
\hline & Posterior & Not used & + & & Infiltrating tumor & 88 & 1 & 0 \\
\hline
\end{tabular}

$\mathrm{GB}=$ glioblastoma; $\mathrm{MUT}=$ mutant; Oligo = oligodendroglioma; $\mathrm{WT}=$ wildtype .

Boldface type indicates the overall classification of each biopsy (tumor or normal).

${ }^{*}$ Sample staining positive for IDH-R132H mutation (+) or not (-).

$\dagger$ Given as a percentage of cells per field of view.

$\ddagger$ Number of spectra after preprocessing.

of 14 biopsies with infiltrated tumor showing fluorescence. At the limit of the resection, where there is a low level of tumor infiltration, the decision regarding further resection balanced against increased risk of neurological disability is most critical. It is at this limit where interrogation with Raman spectroscopy, likely with an intraoperative probebased device, would have application, with our data suggesting that this would give a significantly better tumor predictive value than 5-ALA-induced fluorescence. It is perhaps disheartening that in this study most supramaxi- mal resection cavity biopsies were found to have infiltrating tumor, but it should not be surprising, because these are, after all, named "diffuse gliomas" by the WHO. ${ }^{40}$ Despite this, previous research by our group has shown that supramaximal resection in glioblastoma results in a statistically significant increase in progression-free survival compared to gross-total resection, ${ }^{28}$ and a recently published systematic review and meta-analysis comparing gross-total with supramaximal resection concluded, despite the poor quality of available evidence, that supramaximal resection resulted

TABLE 3. Performance of 5-ALA and Raman spectroscopy to detect tumor versus normal brain

\begin{tabular}{lccc}
\hline \multicolumn{1}{c}{ Modality (classification method) } & Sensitivity & Specificity & Accuracy \\
\hline 5-ALA-induced fluorescence & $0.07(0.02-0.34)$ & $1.00(0.29-1.00)$ & $0.24(0.07-0.50)$ \\
\hline Raman spectroscopy & & & \\
\hline Overall biopsy classification, either including or excluding oligos & $1.00(0.77-1.00)$ & $1.00(0.29-1.00)$ & $1.00(0.85-1.00)$ \\
\hline Individual spectra classification & $0.97(0.96-0.98)$ & $0.72(0.67-0.76)$ & $0.93(0.91-0.94)$ \\
\hline Individual spectra classification including oligos & $0.97(0.96-0.98)$ & $0.68(0.64-0.72)$ & $0.91(0.90-0.92)$ \\
\hline Range in parentheses $=95 \% \mathrm{Cl}$. & & &
\end{tabular}


in a 53\% lower risk of mortality and increased median survival of 6.4 months compared to gross-total resection. ${ }^{41}$

The only reported intraoperative use of a Raman probe comes from Jermyn et al., ${ }^{27}$ whose case series consisted of 17 patients with a mix of tumor pathologies. Brain samples were also taken from areas around the tumor bulk and designated by the neuropathologists as either normal brain or infiltrating tumor. Using a boosted trees machine learning algorithm, they were able to distinguish normal brain from both bulk tumor and infiltrating tumor with an accuracy of $92 \%$, sensitivity of $93 \%$, and specificity of $91 \%$. Our study, although using ex vivo Raman spectroscopy analysis of fresh tissue, adds the important comparison between the performance of Raman spectroscopy and that of the current gold standard of 5-ALA-induced fluorescence.

The principal reason that Raman spectroscopy outperforms 5-ALA fluorescence in this study is the higher detection rate, i.e., higher sensitivity for tumor infiltration, with more than $95 \%$ of spectra of infiltrating tumor correctly classified in most biopsy samples. The correct classification of normal brain is less accurate, with between 59\% and $77 \%$ of spectra from samples with no tumor cells correctly classified. In contrast, the classification model built to classify the cavity biopsies showed high sensitivity and specificity on leave-one-patient-out cross-validation. Here, the normal brain spectra came from samples from patients with epilepsy. This likely reflects the fact that the "normal" penumbra of brain around a glioma is only normal as far as the presence of individual tumor cells is concerned but will show peritumoral edema. It should also be noted that only in IDH1-R132H-mutant glioma can a definitive statement on single-cell invasion of the biopsy cavity can be made. In the IDH-wildtype gliomas, no marker exists that absolutely excludes single-cell infiltration: the proliferation marker Ki-67 (MIB-1) will only highlight atypical nuclei that have entered the cell cycle, and migrating glioma cells have not always entered the cell cycle. The use of normal brain tissue from nontumor patients is still valid, and indeed provides the only guarantee that there are no occult tumor cells within the samples. We note that the mean Raman spectra for tumor and normal brain, as derived from the model, have similar peak characteristics and differences to those reported in published data on tumor versus normal brain Raman spectroscopy analysis. ${ }^{22-24,27,42}$

The data presented here show that Raman spectroscopy is more sensitive at detecting tumor, and has a lower falsenegative rate, than 5-ALA-induced fluorescence, but that the false-positive rate using Raman spectroscopy analysis might be problematic. In the Jermyn et al. paper, a specificity of $91 \%$ is reported. ${ }^{27}$ This is an encouragingly high figure, but in practical terms means that just under 1 in 10 areas that are sampled will give a false-positive result, indicating tumor in an area of potentially normal brain. Jermyn et al. explored in more detail the false-negative rate and estimated the threshold of detection for their intraoperative device to be approximately $15 \%$ cancer cell burden, or 17 human cancer cells $/ 0.0625 \mathrm{~mm}^{2}$. An attempt to quantify the false-negative rate must be commended, but we would suggest some caution in the interpretation of these data as the correlation between the area sampled in vivo by the probe and then biopsied and reviewed by neuropathology may not be completely accurate. Moreover, in only 4 of the 14 samples included in this subanalysis was the specific tumor marker IDH1-R132H used, with the remaining samples only evaluated using $\mathrm{H} \& \mathrm{E}$ staining, and thus not having a specific marker for diffuse glioma cells. Future intraoperative studies should include an additional step in the protocol in which, after intraoperative Raman spectroscopy analysis and biopsy, the fresh sample immediately undergoes ex vivo Raman spectroscopy analysis with the probe device and/or an ex vivo Raman microspectrometer. Accurately establishing the threshold for tumor detection by a Raman probe is an essential part of any future work, but the focus should also be on accurately quantifying the false-positive rate. Future intraoperative Raman probe projects require the clinical spectroscopy and neurosurgery communities to agree on what level of accuracy is both technically achievable and clinically useful to allow useful intraoperative decision-making.

\section{Study Limitations and Future Work}

The main limitations of this study are the small number of cases and the ex vivo Raman analysis. The small sample size prevents a more detailed analysis of the data that would ideally include reporting the model performance with each permutation of tumor density and fluorescence. Moreover, it must be noted that the performance of the model is likely to fall as the number of samples increases due to the increase in variability that would be introduced. However, in this study we report a high-performing model that we feel would be robust to such an increase in variability; further studies with significantly greater sample sizes will help clarify this. In vivo studies will have significant challenges, including reduced performance due to blood or fluid in the measurement field, signal loss from the probe fiber, and noise from surrounding light sources. Some of these issues have been addressed by Jermyn et al., ${ }^{27,43}$ and some methodological considerations have been mentioned above. Future intraoperative Raman probe evaluation will require significant collaboration between neurosurgeons and clinical spectroscopists to produce a usable intraoperative device that is highly accurate and user-friendly.

\section{Conclusions}

With this further work it is feasible that Raman spectroscopy could become an important intraoperative tool used in conjunction with 5-ALA to perhaps guide resection beyond the 5-ALA fluorescence boundary where appropriate, allowing for maximal or supramaximal resection and possibly an increase in patient survival.

\section{Acknowledgments}

A clinical research training fellowship was awarded to L.J.L. and was funded by the Cancer Research UK (CRUK) Oxford Centre. The Oxford Brain Bank is supported by funds from the Medical Research Council (MRC), Brains for Dementia Research (BDR; Alzheimer Society and Alzheimer Research UK), and the NIHR Oxford Biomedical Research Centre (to O.A.). The views expressed are those of the authors and not necessarily those of the NHS, the NIHR, or the Department of Health. This work uses data provided by patients and collected by the NHS as 
part of their care and support and would not have been possible without access to these data. The NIHR recognizes and values the role of patient data, securely accessed and stored, in both underpinning and leading to improvements in research and care. Spectroscopic equipment and data analysis software were loaned by Renishaw plc.

\section{References}

1. Brown TJ, Brennan MC, Li M, et al. Association of the extent of resection with survival in glioblastoma: a systematic review and meta-analysis. JAMA Oncol. 2016;2(11):1460-1469.

2. D'Amico RS, Englander ZK, Canoll P, Bruce JN. Extent of resection in glioma-a review of the cutting edge. World Neurosurg. 2017:103:538-549.

3. Jenkinson MD, Barone DG, Bryant A, et al. Intraoperative imaging technology to maximise extent of resection for glioma. Cochrane Database Syst Rev. 2018;1:CD012788.

4. Senft C, Bink A, Franz K, et al. Intraoperative MRI guidance and extent of resection in glioma surgery: a randomised, controlled trial. Lancet Oncol. 2011;12(11):997-1003.

5. Stummer W, Pichlmeier U, Meinel T, et al. Fluorescenceguided surgery with 5-aminolevulinic acid for resection of malignant glioma: a randomised controlled multicentre phase III trial. Lancet Oncol. 2006;7(5):392-401.

6. Brain tumours (primary) and brain metastases in adults. NICE guideline [NG99]. National Institute for Health and Care Excellence (NICE); 2018. Accessed June 29, 2020. https://www.nice.org.uk/guidance/NG99

7. Lau D, Hervey-Jumper SL, Chang S, et al. A prospective Phase II clinical trial of 5-aminolevulinic acid to assess the correlation of intraoperative fluorescence intensity and degree of histologic cellularity during resection of high-grade gliomas. J Neurosurg. 2016;124(5):1300-1309.

8. Roberts DW, Valdés PA, Harris BT, et al. Coregistered fluorescence-enhanced tumor resection of malignant glioma: relationships between $\delta$-aminolevulinic acid-induced protoporphyrin IX fluorescence, magnetic resonance imaging enhancement, and neuropathological parameters. Clinical article. J Neurosurg. 2011;114(3):595-603.

9. Valdés PA, Kim A, Brantsch M, et al. $\delta$-aminolevulinic acid-induced protoporphyrin IX concentration correlates with histopathologic markers of malignancy in human gliomas: the need for quantitative fluorescence-guided resection to identify regions of increasing malignancy. Neuro Oncol. 2011;13(8):846-856.

10. Valdés PA, Leblond F, Kim A, et al. Quantitative fluorescence in intracranial tumor: implications for ALA-induced PpIX as an intraoperative biomarker. J Neurosurg. 2011; 115(1):11-17.

11. Stummer W, Tonn JC, Goetz C, et al. 5-Aminolevulinic acid-derived tumor fluorescence: the diagnostic accuracy of visible fluorescence qualities as corroborated by spectrometry and histology and postoperative imaging. Neurosurgery. 2014;74(3):310-320.

12. Hollon TC, Lewis S, Pandian B, et al. Rapid intraoperative diagnosis of pediatric brain tumors using stimulated Raman histology. Cancer Res. 2018;78(1):278-289.

13. Broadbent B, Tseng J, Kast R, et al. Shining light on neurosurgery diagnostics using Raman spectroscopy. J Neurooncol. 2016;130(1):1-9.

14. Hollon T, Lewis S, Freudiger CW, et al. Improving the accuracy of brain tumor surgery via Raman-based technology. Neurosurg Focus. 2016;40(3):E9.

15. Valdés PA, Roberts DW, Lu F-K, Golby A. Optical technologies for intraoperative neurosurgical guidance. Neurosurg Focus. 2016;40(3):E8.

16. Gajjar K, Heppenstall LD, Pang W, et al. Diagnostic segregation of human brain tumours using Fourier-transform infra- red and/or Raman spectroscopy coupled with discriminant analysis. Anal Methods. 2012;5:89-102.

17. Krafft C, Sobottka SB, Schackert G, Salzer R. Near infrared Raman spectroscopic mapping of native brain tissue and intracranial tumors. Analyst (Lond). 2005;130(7): 1070-1077.

18. Krafft C, Neudert L, Simat T, Salzer R. Near infrared Raman spectra of human brain lipids. Spectrochim Acta A Mol Biomol Spectrosc. 2005;61(7):1529-1535.

19. Krafft C, Belay B, Bergner N, et al. Advances in optical biopsy-correlation of malignancy and cell density of primary brain tumors using Raman microspectroscopic imaging. Analyst (Lond). 2012;137(23):5533-5537.

20. Köhler M, Machill S, Salzer R, Krafft C. Characterization of lipid extracts from brain tissue and tumors using Raman spectroscopy and mass spectrometry. Anal Bioanal Chem. 2009;393(5):1513-1520.

21. Auner AW, Kast RE, Rabah R, et al. Conclusions and data analysis: a 6-year study of Raman spectroscopy of solid tumors at a major pediatric institute. Pediatr Surg Int. 2013; 29(2):129-140.

22. Kalkanis SN, Kast RE, Rosenblum ML, et al. Raman spectroscopy to distinguish grey matter, necrosis, and glioblastoma multiforme in frozen tissue sections. J Neurooncol. 2014; 116(3):477-485.

23. Kast RE, Auner GW, Rosenblum ML, et al. Raman molecular imaging of brain frozen tissue sections. J Neurooncol. 2014; 120(1):55-62.

24. Kast R, Auner G, Yurgelevic S, et al. Identification of regions of normal grey matter and white matter from pathologic glioblastoma and necrosis in frozen sections using Raman imaging. J Neurooncol. 2015;125(2):287-295.

25. Aguiar RP, Silveira L Jr, Falcão ET, et al. Discriminating neoplastic and normal brain tissues in vitro through Raman spectroscopy: a principal components analysis classification model. Photomed Laser Surg. 2013;31(12):595-604.

26. Livermore LJ, Isabelle M, Bell IM, et al. Rapid intraoperative molecular genetic classification of gliomas using Raman spectroscopy. Neurooncol Adv. 2019;1(1):vdz008.

27. Jermyn M, Mok K, Mercier J, et al. Intraoperative brain cancer detection with Raman spectroscopy in humans. Sci Transl Med. 2015;7(274):274ra19.

28. Livermore LJ, Williams S, Clifton L, et al. Functionally guided supramaximal resection of IDH-wildtype glioblastomas and the effect on progression free survival. Neuro Oncol. 2018;20(suppl_5):v346-v347.

29. Stummer W, Stocker S, Novotny A, et al. In vitro and in vivo porphyrin accumulation by $\mathrm{C} 6$ glioma cells after exposure to 5-aminolevulinic acid. J Photochem Photobiol B. 1998;45(23):160-169.

30. Stummer W, Stepp H, Wiestler OD, Pichlmeier U. Randomized, prospective double-blinded study comparing 3 different doses of 5-aminolevulinic acid for fluorescence-guided resections of malignant gliomas. Neurosurgery. 2017;81(2): 230-239.

31. Cahill DP, Sloan AE, Nahed BV, et al. The role of neuropathology in the management of patients with diffuse low grade glioma: a systematic review and evidence-based clinical practice guideline. J Neurooncol. 2015;125(3):531-549.

32. Afseth NK, Kohler A. Extended multiplicative signal correction in vibrational spectroscopy, a tutorial. Chemom Intell Lab Syst. 2012;117:92-99.

33. Movasaghi Z, Rehman S, Rehman IU. Raman spectroscopy of biological tissues. Appl Spectrosc Rev. 2007;42(5):493-541.

34. Altman DG, Machin D, Bryant TN, Gardner MJ, eds. Statistics With Confidence. 2nd ed. BMJ Books; 2000.

35. Mercaldo ND, Lau KF, Zhou XH. Confidence intervals for predictive values with an emphasis to case-control studies. Stat Med. 2007;26(10):2170-2183. 
36. Kim S, Lee W. Does McNemar's test compare the sensitivities and specificities of two diagnostic tests? Stat Methods Med Res. 2017;26(1):142-154.

37. Petrie A, Sabin C. Medical Statistics at a Glance. 3rd ed. Wiley-Blackwell; 2009.

38. Krafft C, Steiner G, Beleites C, Salzer R. Disease recognition by infrared and Raman spectroscopy. J Biophotonics. 2009; 2(1-2):13-28.

39. Hollon TC, Pandian B, Adapa AR, et al. Near real-time intraoperative brain tumor diagnosis using stimulated Raman histology and deep neural networks. Nat Med. 2020;26(1): 52-58.

40. Louis D, Ohgak H, Wiestler O, et al. WHO Classification of Tumours of the Central Nervous System. 4th ed. WHO; 2016.

41. Incekara F, Koene S, Vincent AJPE, et al. Association between supratotal glioblastoma resection and patient survival: a systematic review and meta-analysis. World Neurosurg. 2019;127:617-624.e2.

42. Meyer T, Bergner N, Bielecki C, et al. Nonlinear microscopy, infrared, and Raman microspectroscopy for brain tumor analysis. J Biomed Opt. 2011;16(2):021113.

43. Desroches J, Jermyn M, Mok K, et al. Characterization of a Raman spectroscopy probe system for intraoperative brain tissue classification. Biomed Opt Express. 2015;6(7):23802397.

\section{Disclosures}

Spectroscopic instrumentation and data analysis software were loaned by Renishaw plc. M.I. and I.M.B. are full-time employees of Renishaw spectroscopic division. Renishaw did not provide any additional funding for the project or individually to the authors.

\section{Author Contributions}

Conception and design: Livermore, Isabelle, Bell, Ansorge. Acquisition of data: Livermore, Edgar, Stacey, Ansorge. Analysis and interpretation of data: Livermore, Isabelle, Bell. Drafting the article: Livermore. Critically revising the article: Isabelle, Bell, Edgar, Voets, Stacey, Ansorge, Vallance, Plaha. Reviewed submitted version of manuscript: Livermore, Isabelle, Edgar, Voets, Stacey, Ansorge, Vallance, Plaha. Approved the final version of the manuscript on behalf of all authors: Livermore. Statistical analysis: Livermore. Administrative/technical/material support: Livermore, Isabelle, Bell, Ansorge, Vallance. Study supervision: Isabelle, Bell, Ansorge, Vallance, Plaha.

\section{Correspondence}

Laurent J. Livermore: Oxford University Hospitals NHS Foundation Trust, John Radcliffe Hospital, Oxford, UK. james.livermore@ouh.nhs.uk. 